\title{
Cuidados de enfermería percibidos por pacientes y su relación con la hospitalización
}

\author{
Nursing care perceived by patients and its relationship with hospitalization \\ Arredondo-Calderón, Stephany Paola ${ }^{1}$ \\ Moreno-Arias, Gabriela Pilar ${ }^{1}$ \\ Ortiz-Montalvo, Yonathan Josué ${ }^{2}$
}

\begin{abstract}
Resumen
Objetivo: determinar la relación entre los factores sociodemográficos y hospitalarios con la percepción de los pacientes sobre los cuidados de enfermería. Materiales y métodos: el estudio fue descriptivo correlacional con diseño transversal. La población estuvo conformada por 50 pacientes hospitalizados, de ambos sexos y mayores de 18 años. La variable principal fue la percepción del paciente sobre el cuidado que recibe de la enfermera y medida a través de un cuestionario. La misma fue clasificada en cuatros dimensiones: biológica, sociocultural, emocional y espiritual. Se utilizó una ficha para obtener datos sociodemográficos y de hospitalización. Para el análisis estadístico inferencial se emplearon las pruebas de correlación de Pearson, T de Student y ANOVA. Resultados: la media de la percepción del paciente sobre el cuidado de enfermería fue 44.72. Además, se evidenció una relación entre la variable recomendación del hospital $(\mathrm{p}=0.001)$ y lugar de procedencia $(\mathrm{p}=0.001)$ con la percepción del paciente sobre el cuidado de su enfermero y las dimensiones biológica, sociocultural, emocional. De igual modo, hubo una relación entre el tipo de servicio y la dimensión espiritual $(\mathrm{p}=0.01)$ y, por último, el ingreso al hospital con la dimensión biológica $(\mathrm{p}=0.03)$. Conclusión: los pacientes que percibieron de manera positiva los cuidados de enfermería son los que han ingresado por primera vez al hospital, son hospitalizados en el servicio cirugía, recomiendan el hospital y proceden de provincias. Ante estos hallazgos se incentiva que en los hospitales se evalué la calidad de cuidados de enfermería para lograr una mayor satisfacción del paciente.
\end{abstract}

Palabras clave: Atención de enfermería; Percepción; Pacientes (Fuente: DeCS).

\begin{abstract}
Objective: to determine the relationship between sociodemographic and hospital factors with patients' perception of nursing care. Materials and methods: the study was descriptive correlational with a cross-sectional design. The population consisted of 50 hospitalized patients, of both sexes and over 18 years of age. The main variable was the patient's perception of the care they receive from the nurse and measured through a questionnaire. It was classified into four dimensions: biological, sociocultural, emotional, and spiritual. A card was used to obtain sociodemographic and hospitalization data. For statistical inferential analysis, Pearson's correlation tests, Student's T test and ANOVA were used. Results: the mean of the patient's perception of nursing care was 44.72. In addition, a relationship between the hospital recommendation variable $(p=0.001)$ and place of origin $(p=0.001)$ with the patient's perception of the care of their nurse and the biological, sociocultural, emotional dimensions was evident. Similarly, there was a relationship between the type of service and the spiritual dimension $(\mathrm{p}=0.01)$ and, finally, admission to the hospital with the biological dimension $(\mathrm{p}=0.03)$. Conclusion: the patients who positively perceived nursing care are those who have entered the hospital for the first time, are hospitalized in the surgery department, recommend the hospital, and come from the provinces. Given these findings, it is encouraged that hospitals evaluate the quality of nursing care to achieve greater patient satisfaction.
\end{abstract}

Keys words: Nursing Care; Perception; Patients (Source: DeCS).

Para citar:

Arredondo SP, Moreno GP, Ortiz YJ. Cuidados de enfermería percibidos por pacientes y su relación con la hospitalización. CASUS. 2020;5(1):41- 47.

DOI: $0.35626 /$ casus. 1.2020 .244

\author{
${ }^{1}$ Hospital Cayetano Heredia. \\ 2 Facultad de Ciencias de la Salud. Universidad Católica Sedes \\ Sapientiae \\ Correo electrónico: esthef9713@gmail.com
}

Fecha de recepción: 19-11-19

Fecha de envío a pares: 25-11-19

Fecha de aprobación por pares: 19-04-20

Fecha de aceptación: 25-04-20 


\section{INTRODUCCIÓN}

El cuidado ha sido el núcleo del personal de enfermería que ha luchado durante el tiempo por representar la dignidad y los valores humanos en sus prácticas (1) Según Jean Watson el nuevo nivel de atención se basa en teorías en evolución respecto a la conciencia de las dimensiones humanas, nuevas formas de pensar y actuando sobre las relaciones auténticas de comunicación, confianza y significado subjetivo para el mantenimiento de la salud y resultados curativos del paciente (2).

Es por ello, que la enfermería es considerada la ciencia y arte del cuidado y su atención esta dirigida desde una perspectiva biopsicosocial y espiritual con cuidados oportunos de calidad. Los cuales, no solo se suplen las necesidades físicas del paciente, sino que busca que perciban un mejor cuidado (3, 4). Sin embargo, pese a que el cuidado es la esencia de la práctica de enfermería, la atención de enfermería convencional se reconoce como inadecuada en relación con la salud holística, procesos de cuidados relacionales (2). Incluso, la tecnología y la economía han causado preocupaciones de naturaleza ética en las prácticas de enfermería (2).

Se reporta, añadido a lo anterior, la existencia de nosocomios donde el personal de enfermería no brinda un cuidado adecuado al paciente y este no se siente a gusto (5). Asimismo, un estudio realizado a nivel nacional reportó que la mayoría de los pacientes adultos mayores de un hospital público de Lima percibió desfavorablemente el cuidado de enfermería y ello varía según tipo de hospital, números de ingresos, entre otras características sociodemográficas del paciente (6).

Es de suma importancia considerar la percepción del paciente porque es un proceso donde él adquiere conciencia de lo que ocurre en su alrededor; va más allá de una imagen del mundo proporcionado por los sentidos. Se debe considerar que una situación dada es experimentada de manera única por cada individuo ya sea de forma positiva o negativa (7). Este fenómeno podría estar relacionado al perfil sociodemográfico y clínico hospitalario de los pacientes (6). Sin embargo, a menudo no se investigan o se informan menos en la literatura.

En tal sentido, cobra relevancia no solo percibir los procesos terapéuticos o conocimientos del profesional de enfermería, sino que también el respeto, la empatía, confianza, etc. $(7,8)$ Lamentablemente, se ha vuelto usual que el usuario perciba a la enfermera como alguien que brinda un mal trato respecto a sus cuidados, es por ello que, la percepción debe ser considerada una herramienta fundamental para conocer la calidad de la atención del enfermero y la satisfacción del paciente (9). Por esta razón, el objetivo del presente estudio fue determinar la relación entre los factores sociodemográficos y hospitalarios con la percepción de los pacientes sobre sus cuidados de enfermería en un Hospital público de Lima Norte en el año 2017.

\section{MATERIALES Y MÉTODOS}

El presente estudio fue descriptivo correlacional de corte transversal. La población fue de 50 pacientes de un hospital nacional de Lima Norte, mayor o igual a 18 años. No se consideró el tamaño de la muestra porque se tuvo en cuenta a toda la población. Asimismo, se incluyeron a los pacientes de ambos sexos de servicios de hospitalización general (Medicina y Cirugía) y se excluyeron a los que no desearon participar en el estudio.

La variable principal fue la "Percepción del paciente sobre el cuidado que recibe de la enfermera" (PCE), definida como un conjunto de procesos mentales donde las personas son capaces de seleccionar, organizar e interpretar la información sobre pensamientos y sentimientos (7). El cuestionario fue un instrumento integrado por 22 ítems, validado por jueces expertos con un coeficiente de Alpha de Cronbach de 0.75 (10). El cuestionario consta de una escala tipo Likert: de siempre ( 3 puntos), a veces (dos puntos) y nunca (1 punto) y con un puntaje de 0 a 66 .

Asimismo, estuvo compuesta por cuatro dimensiones: dimensión biológica (se basa en la percepción del paciente sobre los cuidados de enfermería referentes a las necesidades básicas del 
Tabla 1. Descripción de las variables de la muestra

\begin{tabular}{|c|c|}
\hline & n $(\%)$ \\
\hline \multicolumn{2}{|l|}{ Sexo } \\
\hline Femenino & $28(56.0)$ \\
\hline Masculino & $22(44.0)$ \\
\hline \multicolumn{2}{|l|}{ Servicio } \\
\hline Medicina & $25(50.0)$ \\
\hline Cirugía & $25(50.0)$ \\
\hline Edad $($ media \pm DS $)$ & $48.3 \pm 14.8$ \\
\hline \multicolumn{2}{|l|}{ Estado civil } \\
\hline Soltera & $12(24.4)$ \\
\hline Casada & $6(12.2)$ \\
\hline Conviviente & $26(53.6)$ \\
\hline Separada & $5(10.2)$ \\
\hline \multicolumn{2}{|l|}{ Tiene hijos } \\
\hline Sí & $44(88.0)$ \\
\hline No & $6(12.0)$ \\
\hline \multicolumn{2}{|l|}{ Lugar de procedencia } \\
\hline Lima & $34(68.0)$ \\
\hline Provincia & $16(32.0)$ \\
\hline \multicolumn{2}{|l|}{ Nivel educativo } \\
\hline Primaria & $6(12.0)$ \\
\hline Secundaria & $39(78.0)$ \\
\hline Técnico & $4(8.0)$ \\
\hline Universitario & $1(2.0)$ \\
\hline \multicolumn{2}{|l|}{ Trabaja actualmente } \\
\hline Sí & $27(54.0)$ \\
\hline No & $23(46.0)$ \\
\hline \multicolumn{2}{|l|}{ Ingresos al hospital } \\
\hline Una vez & $33(66.0)$ \\
\hline Más veces & $7(34.0)$ \\
\hline \multicolumn{2}{|l|}{ Conoce el nombre de la enfermera } \\
\hline Sí & $1(2.0)$ \\
\hline No & $9(98.0)$ \\
\hline \multicolumn{2}{|l|}{ Recomendaría el hospital } \\
\hline Sí & $36(72.0)$ \\
\hline No & $14(28.0)$ \\
\hline D. biológica (media \pm DE) & $14.2 \pm 3.1$ \\
\hline D. socio cultural (media \pm DE) & $16.1 \pm 2.5$ \\
\hline D. espiritual (media \pm DE) & $6.6 \pm 2.2$ \\
\hline D. emocional (media \pm DE) & $7.8 \pm 1.8$ \\
\hline $\begin{array}{l}\text { Percepción de los pacientes } \\
\text { sobre sus cuidados (media } \pm \\
\text { DE) }\end{array}$ & $44.72 \pm 6.49$ \\
\hline
\end{tabular}

ser humano como alimentación, higiene, eliminación, sueño y otras). Dimensión sociocultural (conductas de atención, valores y creencias basados en necesidades culturales y sociales de los pacientes). Dimensión espiritual (preocupación por aspecto espiritual y respeto de creencias religiosas) y la dimensión emocional (está relacionada con los sentimientos y emociones del paciente durante estancia hospitalaria). Las dos primeras dimensiones tuvieron 7 preguntas y un
Tabla 2. Relación entre las variables sociodemográficas con la percepción de los pacientes sobres sus cuidados

\begin{tabular}{|c|c|c|}
\hline & \multicolumn{2}{|c|}{$\begin{array}{l}\text { Percepción de los } \\
\text { pacientes sobre } \\
\text { sus cuidados }\end{array}$} \\
\hline Sexo & & 0.96 \\
\hline Femenino & $44.7(7.5)$ & \\
\hline Masculino & $44.8(5.2)$ & \\
\hline Servicio & & 0.80 \\
\hline Medicina & $44.96(8.8)$ & \\
\hline Cirugía & $44.5(2.8)$ & \\
\hline Edad (r) & 0.289 & 0.04 \\
\hline Tiene hijos & & 0.96 \\
\hline $\mathrm{Si}_{1}$ & $44.7(6.7)$ & \\
\hline No & $44.8(5.5)$ & \\
\hline Estado civil & & 0.47 \\
\hline Soltera & $42.8(9.4)$ & \\
\hline Casada & $46.8(2.7)$ & \\
\hline Conviviente & $44.2(5.5)$ & \\
\hline Separada & $47(2.1)$ & \\
\hline Lugar de procedencia & & 0.00 \\
\hline Lima & $42.97(6.5)$ & \\
\hline Provincia & $48.4(4.8)$ & \\
\hline Nivel educativo & & 0.52 \\
\hline Primaria & $44(5.1)$ & \\
\hline Secundaria & $45.3(6.7)$ & \\
\hline Técnico & $41.8(6.9)$ & \\
\hline Universitario & $38(0.0)$ & \\
\hline Trabaja actualmente & & 0.33 \\
\hline Sí & $45.7(8.6)$ & \\
\hline No & $43.9(3.94)$ & \\
\hline Ingresos al hospital & & 0.18 \\
\hline Una vez & $45.6(5.4)$ & \\
\hline Más veces & $43(8.1)$ & \\
\hline Recomendaría & & \\
\hline hospital & & 0.00 \\
\hline Si & $46.3(5.7)$ & \\
\hline No & $40.7(6.8)$ & \\
\hline
\end{tabular}

puntaje de 0 a 21 , y las dos últimas solo 4 preguntas $\mathrm{y}$ un puntaje de 0 a 12. La variable y sus dimensiones se consideraron bajo su naturaleza cuantitativa.

Respecto a las variables sociodemográficas y clínicas se empleó una ficha de datos que buscó obtener información sobre: sexo, variable cualitativa dicotómica (femenino y masculino); edad, variable cuantitativa; estado civil, variable cualitativa (soltero(a), casado(a), conviviente, separado(a)); tiene hijos, variable cualitativa dicotómica (sí y no); número de hijos, variable cuantitativa; lugar de procedencia, variable 
Tabla 3. Relación entre las variables sociodemográficas con las dimensiones de la percepción de los pacientes sobres sus cuidados

\begin{tabular}{|c|c|c|c|c|c|c|c|c|}
\hline & \multicolumn{2}{|c|}{ Dimensión Biológica } & \multicolumn{2}{|c|}{$\begin{array}{c}\text { Dimensión } \\
\text { Sociocultural }\end{array}$} & \multicolumn{2}{|c|}{ Dimensión Espiritual } & \multicolumn{2}{|c|}{$\begin{array}{l}\text { Dimensión } \\
\text { Emocional }\end{array}$} \\
\hline & Media (DE) & $\mathbf{p}$ & Media (DE) & $\mathbf{p}$ & Media (DE) & $\mathbf{p}$ & Media (DE) & $\mathbf{p}$ \\
\hline \multicolumn{9}{|l|}{ Sexo } \\
\hline Femenino & $14.4(3.4)$ & 0.69 & $16.2(2.8)$ & 0.77 & $6.6(2.2)$ & 0.31 & $7.8(2.0)$ & 0.96 \\
\hline Masculino & $14(2.6)$ & & $16(2.2)$ & & $7(2.3)$ & & $7.8(1.3)$ & \\
\hline \multicolumn{9}{|l|}{ Servicio } \\
\hline Medicina & $14.5(4.0)$ & 0.52 & $16.7(3.2)$ & 0.10 & $5.8(2.7)$ & 0.00 & $8(2.2)$ & 0.34 \\
\hline Cirugía & $13.9(1.6)$ & & $15.5(1.5)$ & & $7.5(1.0)$ & & $7.5(1.1)$ & \\
\hline Edad (r) & 0.322 & 0.02 & 0.144 & 0.319 & 0.00 & 0.949 & 0.263 & 0.06 \\
\hline \multicolumn{9}{|l|}{ Tiene hijos } \\
\hline Sí & $14.2(3.1)$ & 0.87 & $16.1(2.6)$ & 0.9 & $6.5(2.3)$ & 0.42 & $7.8(1.8)$ & 0.70 \\
\hline No & $14(3.2)$ & & $16(1.9)$ & & $7.3(1.0)$ & & $7.5(0.8)$ & \\
\hline \multicolumn{9}{|l|}{ Estado civil } \\
\hline Soltera & $13.4(4.2)$ & 0.48 & $15.8(2.10)$ & 0.82 & $6.3(2.1)$ & 0.88 & $7.3(2.2)$ & 0.28 \\
\hline Casada & $14.5(0.5)$ & & $16.5(2.4)$ & & $7(1.8)$ & & $8.8(1.2)$ & \\
\hline Conviviente & $13.9(2.3)$ & & $15.9(2.3)$ & & $6.8(2.8)$ & & $7.6(1.5)$ & \\
\hline Separada & $15.8(3.8)$ & & $16.8(2.8)$ & & $6.2(3.8)$ & & $8.2(1.9)$ & \\
\hline \multicolumn{9}{|c|}{ Lugar de procedencia } \\
\hline Lima & $13.4(2.6)$ & 0.01 & $15.6(2.6)$ & 0.05 & $6.4(2.5)$ & 0.36 & $7.5(1.7)$ & 0.09 \\
\hline Provincia & $15.9(2.8)$ & & $17.1(2.2)$ & & $7.1(1.4)$ & & $8.4(1.7)$ & \\
\hline \multicolumn{9}{|l|}{ Nivel educativo } \\
\hline Primaria & $15.5(3.4)$ & 0.28 & $15(1.7)$ & 0.58 & $6.2(3.3)$ & 0.82 & $7.3(1.2)$ & 0.67 \\
\hline Secundaria & $14.3(2.9)$ & & $16.4(2.7)$ & & $6.7(2.1)$ & & $7.9(1.9)$ & \\
\hline Técnico & $12.3(3.9)$ & & $15.5(1)$ & & $7(1.4)$ & & $7(1.2)$ & \\
\hline Universitario & $11(0)$ & & $15(0)$ & & $5(0)$ & & $7(0)$ & \\
\hline \multicolumn{9}{|c|}{ Trabaja actualmente } \\
\hline Sí & $13.10(2.4)$ & 0.56 & $15.6(1.8)$ & 0.11 & $6.7(2.0)$ & 0.83 & $7.6(1.3)$ & 0.57 \\
\hline No & $14.5(3.7)$ & & $16.7(3.1)$ & & $6.6(2.4)$ & & $7.9(2.2)$ & \\
\hline \multicolumn{9}{|c|}{ Ingresos al hospital } \\
\hline Una vez & $14.9(2.8)$ & 0.03 & $16.2(2.3)$ & 0.64 & $6.6(2.2)$ & 0.78 & $7.9(1.9)$ & 0.41 \\
\hline Más veces & $12.9(3.0)$ & & $15.9(3.0)$ & & $6.8(2.2)$ & & $7.5(1.5)$ & \\
\hline \multicolumn{9}{|c|}{$\begin{array}{l}\text { Recomendaría el } \\
\text { hospital }\end{array}$} \\
\hline Sí & $14.8(3.1)$ & 0.02 & $16.7(2.5)$ & 0.01 & $6.7(2.3)$ & 0.68 & $8.1(1.7)$ & 0.04 \\
\hline No & $12.6(2.4)$ & & $14.7(2.1)$ & & $6.4(2.1)$ & & $6.9(1.5)$ & \\
\hline
\end{tabular}

cualitativa dicotómica (Lima y provincia); nivel de estudios, variable cualitativa ( secundaria, técnico, universitario); ingresos al hospital, variable cualitativa dicotómica (una y más veces); ¿Conoce el nombre de la enfermera que lo atendía cada turno?, variable cualitativa dicotómica (sí y no) y recomendaría el hospital a otras personas por la atención que brinda el personal de Enfermería, variable cualitativa dicotómica (sí y no).

Los datos del estudio se analizaron mediante el programa estadístico Stata 14. Para la realización del análisis descriptivo se calcularon promedios para las variables cuantitativas y porcentajes para las variables cualitativas. De igual modo se utilizó la prueba de Kolmogorov Smirnov para comprobar la normalidad de la variable. Para el análisis bivariado, se emplearon las pruebas de correlación de Pearson, T de Student y ANOVA. Significancia estadística fue menor o igual a 0.05 .

Para la recolección de los datos el estudio contó con la aprobación del comité de ética de la Universidad Católica Sedes Sapientiae. Del mismo modo, se obtuvo el consentimiento informado y se respetó la confidencialidad de la información y el anonimato los participantes.

\section{RESULTADOS}

Los resultados reportaron que del total de los 
participantes hospitalizados la media de la edad fue de 48.3 años, el $56.0 \%$ fue del sexo femenino, el $53.6 \%$ refirió ser conviviente, el $88 \%$ con hijos, el $68 \%$ fue procedente de Lima y el $32 \%$ de provincia. Asimismo, el $98 \%$ de encuestados no conoció el nombre de la enfermera(o) que les brindó sus cuidados y el $66 \%$ ingresó por primera vez al hospital. Respecto a la media de la variable principal percepción del paciente sobre el cuidado que recibe de la enfermera fue 44.72 (de un rango de 0 a 66) y el mayor promedio de las dimensiones fueron sociocultural (media $=16.1$ de un rango de 0 a 21) y emocional (media $=7.8$ de un rango de $0 \mathrm{a}$ 12). Para más información se consulte la tabla 1.

En las tablas 2 y 3 se observa una correlación baja pero significativa entre la edad y la variable principal: percepción del paciente sobre el cuidado que recibe de la enfermera $(\mathrm{r}=0.2 ; \mathrm{p}=0.04)$ y la dimensión biológica $(\mathrm{r}=0.3 ; \mathrm{p}=0.02)$, asimismo, hubo relación entre el lugar de procedencia y la variable principal $(\mathrm{p}=0.001)$ y las dimensiones biológica $(\mathrm{p}=0.01)$ y sociocultural $(\mathrm{p}=0.05)$, es decir los pacientes que procedieron de provincias tuvieron un mayor promedio de PCE (media=48.4) y de dichas dimensiones, de igual modo, se evidenció una relación entre recomendaría el hospital y la variable principal $(\mathrm{p}=0.001)$ y las dimensiones biológica $(\mathrm{p}=0.02)$, sociocultural $(\mathrm{p}=0.01)$ y emocional $(\mathrm{p}=0.04)$, donde los pacientes que recomendarían el hospital a personas por la atención que brinda el personal de Enfermería tienen una mayor promedio de PCE $($ media $=46.3)$ y de aquellas dimensiones.

Por otro lado, en la tabla 3 , solo se observa una relación entre ingresos al hospital con la dimensión biológica $(\mathrm{p}=0.03$ ), en el cual los pacientes que tuvieron un solo ingreso al hospital presentaron un mayor promedio de esta dimensión (14.9) en comparación de los que tuvieron más ingresos (media=12.9). Por último, se encontró asociación entre servicio y la dimensión espiritual $(\mathrm{p}=0.01)$ donde los que tuvieron un mayor promedio de esta dimensión fueron los hospitalizados en el servicio cirugía (media=7.5).

\section{DISCUSIÓN}

Los resultados principales mostraron que existe relación entre la variable recomendación al hospital y lugar de procedencia con la percepción del paciente sobre el cuidado que recibe de la enfermera $\mathrm{y}$ las dimensiones biológica, sociocultural y emocional. De igual modo, hubo una relación entre el tipo de servicio y la dimensión espiritual y, por último, entre el ingreso al hospital y la dimensión biológica.

En el estudio se observó que las personas que recomendaron el hospital a terceras personas por la atención que brinda el personal de Enfermería tuvieron una mayor percepción sobre sus cuidados que recibió por parte de su enfermera y en las dimensiones: biológica, sociocultural y emocional. Mientras que los que no recomendaron el hospital el promedio de la PCE y de sus dimensiones fue menor. Estudios realizados en los últimos cinco años en España reportaron que la mayoría de los pacientes con enfermedades crónicas recomendarían sin dudarlo el hospital por los cuidados brindados por los enfermeros $(11,12)$. Es importante considerar que la dimensión biológica, que abarca las patologías crónicas y necesidades fisiológicas de los pacientes, concientiza al personal de enfermería de la necesidad de un cuidado humanizado y holístico (12). Asimismo, los pacientes que refieren tener un alto grado de empatía con el enfermero perciben que el enfermero interactúa con él, se preocupa por conocer sus necesidades sociales, sentimientos y comportamientos, lo cual conlleva a la recomendación del hospital y, por ende, una mayor percepción de la dimensión sociocultural (13). Además, los pacientes necesitan satisfacer sus necesidades frente a la sensación de carencias emocionales debido al mal estado de salud y estancia hospitalaria que presentan y deben ser considerados por los enfermeros para que ellos perciban positivamente sus cuidados $(13,14)$.

De acuerdo con el estudio, se evidenció que los pacientes procedentes de provincia tuvieron una mayor percepción sobre los cuidados que recibe por parte de su enfermera y en las dimensiones: biológica y sociocultural. En comparación de los procedieron de Lima Metropolitana donde el 
promedio de la PCE y sus dimensiones fue menor. Existe poca literatura sobre este hallazgo e incluso una investigación realizada en Perú reportó que no existe asociación significativa entre ambas variables, pero esto se pudo deber a la poca población del estudio (6). Una posible explicación de este hallazgo es que un paciente procedente de provincia percibe una mejor atención por parte del personal de enfermería y los servicios que brinda el hospital debido a que la situación de los hospitales de las provincias en su mayoría es consideradas dramáticas por la falta de personal de salud, medicamentos e insumos, con tecnología desfasada, etc. y pudieran percibir que la atención de salud es mejor en la capital (15). Por el contrario, los que viven en Lima son conocedores de que en la ciudad hay carencias en los hospitales y de recursos humanos y no suplen sus necesidades biológicas y sociales (15).

Del mismo modo, se halló que los pacientes hospitalizados en el servicio Cirugía tuvieron un mayor promedio de percepción cuidado del enfermero en la dimensión espiritual en comparación con los que estuvieron hospitalizados en el servicio de Medicina. Dos estudios reportaron similares resultados $(6,14)$ Esto es probable ya que en el servicio de Cirugía los pacientes experimentan sentimientos de miedo y temor debido al riesgo quirúrgico que presenta toda operación, por ello se aferran más a Dios antes de la intervención y después de ella (16). Asimismo, presentan una serie de pérdidas como la intimidad, autodeterminación, autocuidado, rutina diaria, confort de hogar, imagen corporal, etc. De acuerdo con lo expuesto, el personal de enfermería, quien está en constante interacción con los pacientes desde su ingreso hasta el alta y ellos, debe considerar la dimensión espiritual porque influye positiva o negativamente en la evolución clínica y en la calidad de vida de los pacientes (8).

El último hallazgo fue que los pacientes que ingresaron por primera vez al hospital presentaron un mayor promedio de percepción de cuidados de enfermería referente a la dimensión biológica, mientras que en los ingresaron más veces tuvieron un menor promedio en dicha dimensión. Solo un estudio similar reportó que los pacientes que se hospitalizaron entre 1 a 5 días tuvieron una percepción favorable del cuidado enfermero. Lo contrario en los que se hospitalizaron por más de 16 días (6). Teniendo en cuenta que la dimensión biológica del paciente que permanece hospitalizado en varias ocasiones puede sesgar sus percepciones como cuando se hospitalizó por primera vez, posiblemente vea de manera negativa porque se siente impotente y su recuperación tarde mucho tiempo en el hospital. Pero, por otro lado, podría acostumbrase mejor a la estancia hospitalaria por la empatía que se establece con el enfermero en el servicio (17).

En cuanto a las limitaciones del diseño de estudio se debe considerar los sesgos de información, porque se trata de un cuestionario autodirigido. Por otro lado, la población es reducida debido a la falta de participación de los pacientes por el estado de salud. Sin embargo, la obtención de información mediante el instrumento empleado podría contribuir para la evaluación de la calidad de atención del personal de enfermería.

\section{CONCLUSIONES}

La media de la variable principal percepción del paciente sobre el cuidado que recibe de la enfermera es 44.72, de un rango de 0 a 66 . Los resultados inferenciales mostraron que hay una relación significativa entre las hospitalizaciones, la cantidad de ingresos al hospital, tipo de servicio y la recomendación del hospital a personas por la atención recibida con los cuidados de enfermería percibidos por los pacientes. Asimismo, existe una relación entre la variable principal con el lugar de procedencia de los encuestados. Estas asociaciones son similares en las dimensiones biológica, sociocultural y emocional. Además, los pacientes que percibieron de manera positiva los cuidados de enfermería son los que tienen un solo ingreso al hospital, son hospitalizados en el servicio cirugía, recomiendan el hospital y proceden de provincias.

Por los hallazgos encontrados se recomienda realizar futuros estudios con muestras amplias tantos en hospitales privados como públicos, incluir otras variables como los días de hospitalización, 
ocupación laboral, antecedentes de enfermedades y con respecto a la variable recomendación se debe ampliar más preguntas para evitar sesgos. De igual manera, se propone implementar, en los servicios de los hospitales, cuestionarios sobre la atención recibida por el enfermero antes de que el paciente salga del alta y una ficha de datos hospitalarios y sociodemográficos, para así evaluar la calidad de cuidados que brinda el personal de enfermería y, de esa manera, proponer estrategias para mejorar y brindar un adecuado cuidado holístico a los pacientes.

\section{REFERENCIAS BIBLIOGRÁFICAS}

1. Rad M, Mirhaghi A, Shomoossi N. Loving and humane care: a missing link in nursing. Nursing and midwifery studies. 2016;5(2).

2. Watson J. Commentary on Shattell M (2004) Nurse-patient interaction: a review of the literature. Journal of Clinical Nursing 13, 714-722. Journal of Clinical Nursing. 2005;14(4):5302.

3. Monje V, Miranda C, Oyarzün G, Seguel P, Flores G. Percepción de cuidado humanizado de enfermería desde la perspectiva de usuarios hospitalizados. Ciencia y enfermería. 2018;24.

4. Zarate RA. La gestión del cuidado de enfermería. Index de enfermería. 2004;13(44-45):42-6.

5. Day RH. Psicología de la percepción humana. LimusaWiley; 1973.

6. Martínez N. Tipo de hospital y percepción del adulto mayor acerca del cuidado humanizado del enfermero. CASUS. Revista de Investigación y Casos en Salud. 2018;3(2):64-71.

7. De Cuidado G. Facultad de Enfermería, Universidad Nacional de Colombia. Dimensiones del cuidado, Santafé de Bogotá Unibiblos. 1998

8. Tous CA, Machado AC, Galindo $\mathrm{AB}$, Pérez LB, Arroyo KG Percepción de los pacientes acerca del cuidado de enfermería en una clínica de sincelejo Colombia. Revista Horizonte de Enfermería. 2020;28(1):7-26

9. Ma CC, Samuels ME, Alexander JW. Factors that influence nurses' job satisfaction. JONA: The Journal of Nursing Administration. 2003;33(5):293-9.

10. Zapata S. Percepción del paciente adulto mayor sobre el cuidado que recibe de enfermera en el servicio de geriatría del hospital Almenara. [Tesis de Licenciatura]. Perú: Escuela de Enfermería Padre Luis Tezza Afiliada a la Universidad Ricardo Palma; 2013.

11. Medina MC. Calidad percibida por el paciente en relación a los cuidados de enfermería en pacientes sometidos a una artroplastia total de rodilla. Revista Enfermería C y L. 2016;8(2):3-9.

12. Toledano L, Carmen M, Luque Cantarero I, Gómez VE, Casas R, Crespo R. La satisfacción del paciente como componente de la calidad en las unidades de diálisis.
Enfermería

Nefrológica. 2016;19(2):125-33.

13. Silva JR, Ramón S, Vergaray S, Palacios V, Partezani R. Percepción del paciente hospitalizado respecto a la atención de enfermería en un hospital público. Enfermería universitaria. 2015;12(2):80-7.

14. Romero E, Contreras IM, Moncada A. Relación entre cuidado humanizado por enfermería con la hospitalización de pacientes. Hacia la Promoción de la Salud. 2016;21(1):26-36

15. Soto A. Barreras para una atención eficaz en los hospitales de referencia del Ministerio de Salud del Perú: atendiendo pacientes en el siglo XXI con recursos del siglo XX. Revista Peruana de Medicina Experimental y Salud Publica. 2019;36:304-11.

16. Canteros MF. Importancia de los aspectos espirituales y religiosos en la atención de pacientes quirúrgicos. Revista chilena de cirugía. 2016;68(3):258-64.

17. Lenis CA, Manrique FG. Calidad del cuidado de enfermería percibida por pacientes hospitalizados. Aquichan. 2015;15(3):413-25. 